Repères $\mathrm{n}^{\circ} 11$, Ecriture et traitement de texte, INRP.

\title{
I'ECRITURE SUR ORDINATEUR ET SES REPRESENTATIONS CHEZ DES ÉLÈVES DE CM2 EN ATELIER INFORMATIQUE
}

Marie-Christine POUDER

URA 1031 CNRS-Université de Paris V

Résumé : A partir de l'analyse du contenu des réponses d'enfants de CM2 à un questionnaire d'informatique, nous évoquons l'expérience que ces enfants ont du traitement de texte ainsi que la représentation qu'ils se font de son utilisation dans la société. Lié à d'autres outils logiciels, le traitement de texte permet le développement d'une communication écrite diversifiée et complexifiée qui utilise vecteurs traditionnels et vecteurs électroniques. Assis au poste de travail de l'ordinateur, plus identifié au journaliste qu'à l'écrivain, l'enfant intériorise les actions successives de la chaîne des métiers de l'écrit.

\section{RÉVOLUTION INFORMATIQUE ?}

Depuis deux décennies la micro-informatique s'est peu à peu introduite dans le champ domestique et dans le champ scolaire. Elle n'est plus l'apanage de spécialistes, chercheurs, industriels ou professionnels des médias. Elle vise à reproduire, à accélérer, et à automatiser des procédures quotidiennes et répétitives comme l'écriture, le dessin, le calcul, liées à des pratiques sociales qui les incluent (courrier, publication assistée, gestion de budget, etc.).

Bien que la politique scolaire soit assez hésitante en ce qui concerne l'âge de l'initiation à l'informatique (théoriquement au cycle primaire ? mais souvent pratiquement au cycle secondaire), la percée des ordinateurs familiaux se confirme avec l'apparition récente dans les supermarchés de configurations multimédias à des prix abordables. Les CD interactifs, les encyclopédies et dictionnaires sur CD ROM, les premiers livres-laser pour enfants constituent désormais un marché en pleine évolution qui va fixer une certaine clientèle devant le complexe écran / clavier. Les jeunes, désormais familiers de la petite lucarne passeront sans doute de plus en plus facilement de l'écran de télévision à l'écran vidéo (d'ailleurs souvent le même) et à l'écran d'ordinateur et, pour quelques-uns déjà, la découverte du clavier inaugure l'accès à l'écriture.

Cette pratique est-elle vraiment révolutionnaire ? Tend-elle à remplacer une pratique plus traditionnelle ou figure-t-elle plutôt une nouvelle forme d'écriture qui, à la suite d'une écriture manuscrite d'abord réservée à une élite, puis de t'écriture imprimée scolaire, va simplement diversifier les conduites avant qu'un

autre bond en avant ne projette les futurs scripteurs sur les réseaux des autoroutes informatiques ? 
Après avoir rappelé la position de quelques précurseurs, nous verrons comment des petits parisiens de CM2 s'accommodent de ces nouvelles technologies.

\subsection{Papert... et les autres, pour un horizon heureux de l'informatique.}

Le temps n'est pas si lointain où S.Papert (1981) mettait tous ses espoirs dans l'enseignement du langage LOGO, sa création, et dans l'accès à l'ordinateur et au traitement de texte pour le plus grand nombre :

"Pour la majorité des enfants, réécrire un texte est une corvée si pénible que le premier jet est aussi la version définitive, de sorte qu'ils n'apprennent jamais cet art de se relire d'un oeil critique. L'accès à un ordinateur capable du traitement de texte apporte là un changement spectaculaire. Le premier jet est composé sur clavier, les corrections sont faciles à faire. La version en cours, $1^{\mathrm{er}}, 2^{\mathrm{èm}}, \mathrm{n}^{\mathrm{ièm}}$ brouillon est toujours propre et nette. J'ai vu un enfant qui détestait rédiger, au point de refuser de le faire, y prendre intensément goût au bout de quelques semaines, une fois initié à l'écriture sur ordinateur ; et la qualité de ses textes s'améliorait rapidement ... Cette image de l'enfant utilisant l'ordinateur comme instrument d'écriture illustre particulièrement bien mon postulat général selon lequel ce qui est bon pour les professionnels est bon pour les enfants."

Cette vision n'était-elle pas trop optimiste ? Il existe après tout bien des manières d'écrire et celle qui est décrite dans cet extrait n'est pas la seule. N'y-a-t-il pas, quinze ans après, des effets de banalisation et d'habituation ?

\subsection{Effets d'une introduction}

Dès les années 80 , certains auteurs américains ont signalé combien les jeunes élèves étaient intéressés par l'apprentissage de l'informatique, combien cette activité pouvait "fidéliser " certaines populations scolaires difficiles et les stimuler intellectuellement (Papert, 1981, Levin cité par Greenfield $1984)$.

Alors que le système scolaire français pensait alors davantage apprentissage de la programmation et enseignement assisté par ordinateur à partir de produits spécialement conçus pour les scolaires, les pédagogues américains observaient déjà le développement de l'utilisation précoce de traitements de texte professionnels. Leurs conclusions étaient alors optimistes.

Ecrire avec un traitement de texte nécessite de conscientiser un certain nombre de procédures qui sont partiellement automatisées dans l'écriture manuelle rapide. Pour l'adulte peu expert, l'écriture sur clavier met l'accent sur l'épellation, l'orthographe, la ponctuation. Le déroulement des menus incite à varier la mise en page, la taille, le style ou la police des caractères et l'aspect visuel du texte prend brusquement plus d'importance.

L'environnement LOGO et le travail par paires qui lui est souvent lié stimulent la conscience de l'écrit et permettent la confrontation des points de vue des jeunes scripteurs en cas de problème d'écriture. Le travail de groupe délivre le mâ̂tre d'un certain nombre de tâches, et le libère pour un travail 
d'aide personnalisée. Dans ce cas, l'enfant n'est pas seulement en interaction avec sa machine dans un projet d'écriture mais il entre en interaction avec (un de) ses pairs à propos d'une production scripturale socialisée et avec le mâ̂tre pour une élucidation de ses besoins. Pour Greenfield, le traitement de texte rend l'acte d'écriture plus visible, moins solitaire, du moins dans le contexte scolaire (Greenfield, 1984).

Pour cet auteur, l'utilisation du traitement de texte n'est pas suffisante en elle-même pour faciliter aux enfants l'accès à une conscientisation, témoignage du plus haut stade d'activité cognitive, et elle ne remplit un rôle véritablement structurant que lorsqu'elle entraîne le/les scripteurs dans des opérations de réécriture.

L'environnement informatique peut aussi jouer un rôle facilitateur en rendant plus ludique l'activité d'écriture, en présentant aux élèves une image valorisée de leur production et en accélérant le processus de diffusion.

D'après S.Papert, l'informatisation croissante de la société conduit à une nouvelle manière de penser :

"Je ne pense pas que l'ordinateur en soi puisse faire quelque chose qu'il serait impossible de faire sans lui, ne serait-ce que parce que l'enfant pourrait l'inventer et interagir avec lui dans son jeu imaginaire ? Evidemment, lorsque l'ordinateur est concret, palpable, il s'intègre davantage dans le comportement. On voit donc grosso modo, les enfants penser en d'autres termes, exactement comme les scientifiques, puisqu'aujourd'hui la pensée psychologique, presque sans exception est colorée par la pensée informatique. " (Entretiens avec Pessis Pasternak, 1990).

L'impact des produits audiovisuels redonne de l'importance aux conduites orales de la langue ainsi qu'aux conduites non-verbales. L'imagination est de plus en plus liée à la mémoire, à la représentation du mouvement et de l'espace et elle ne correspond plus uniquement à l'ancien ordre des choses plus littéraire au sens strict. Les enfants connaissent maintenant les classiques de la littérature enfantine (contes, récits épiques, récits d'aventure, romans ...) en premier lieu par des adaptations animées ou filmées ; ils ont une culture de l'image et le rapport à l'écriture est désormais second. Le rapport image / oral / écrit est en profonde mutation (Pouder, 1993).

\subsection{Transformation de l'écriture?}

P. Levy semble aller plus loin lorsqu'il affirme que l'écriture elle-même est actuellement modifiée dans certains de ces produits :

"Pour ceux qui se servent régulièrement (du traitement de texte), le texte stocké n'est plus considéré comme le but à obtenir mais plutôt comme la matière première, à partir de laquelle ils le retravailleront. Ils ont donc tendance à écrire de façon modulaire, de manière à pouvoir recombiner ces fragments de texte pour une nouvelle utilisation ... (d'où) la conséquence sur le produit final : de moins en moins de synthèses globales mais d'avantage une écriture combinatoire. " (Entretiens avec Pessis Pasternak, 1990)

Cette idée est développée dans son ouvrage Les technologies de l'intelligence : l'avenir de la pensée à l'ère informatique (1990). Pour cet auteur l'informatique est une troisième révolution dans l'humanité après le règne de l'oral et de l'écrit. "En effet on a constaté une transformation 
radicale dans les manières de penser ; en culture orale, on pensait par "situations " alors que grâce à la culture écrite, on réfléchit par catégories. Or avec l'informatisation, on constate l'émergence d'un nouveau type de gestion sociale des connaissances ; on pense à l'aide de procédures formelles et de " modèles computationnels". Ce nouveau paradigme qui va profondément changer notre vision du monde est celui de l'information opérationnelle qui se fonde sur le calcul et sur l'analyse."

Ainsi voyons-nous arriver dans les collèges et les universités des logiciels de génération de texte vecteurs de nouvelles pratiques d'écriture en prise avec la mathématique et l'aléatoire (Colloque de Cerisy, 1991 , La Recherche, 1995)(note 1). "Ces nouveaux produits permettent de plus en plus de combiner lecture et écriture et font concevoir de moins en moins le processus d'écriture comme fluide et linéaire. Les tâches d'écriture sont de plus en plus liées à des tâches de transformation et de restructuration de genres, de cohésion et d'alignement énonciatif, tâches métadiscursives intégrées.

\section{SUR LE TERRAIN DE L'ATELIER INFORMATIQUE}

Nous tirons les données qui suivent d'un questionnaire d'évaluation, en informatique auquel ont répondu, en Juin 1994, 409 enfants (193 filles et 216 garçons) d'une vingtaine d'ateliers informatiques de la ville de Paris. Ce questionnaire clôturait une année d'initiation à l'informatique, avec initiation au traitement de texte, à des logiciels graphiques et à d'autres produits logiciels divers selon les ateliers. Il comprenait trente-cinq questions portant sur des sujets divers comme l'histoire de l'informatique, le raisonnement, les expériences menées dans les ateliers, certaines connaissances techniques (note 2 ) •

Nous nous appuierons sur le corpus écrit des réponses à quatre des trentecinq questions posées, ainsi que sur des dessins afin de cerner ce que peuvent être les représentations du traitement de texte et de l'ordinateur chez ces jeunes élèves scolarisés en CM2.

- $1^{\text {ère }}$ question : a) Ouel projet as-tu réalisé cette année avec la classe à l'atelier informatique ? b) Qu'est-ce que l'ordinateur a rendu possible ?

- $2^{\text {ème }}$ question : Que peux-tu faire avec un logiciel de traitement de texte que tu ne pourrais pas faire avec une machine à écrire ? (note 3)

- $3^{\text {ème }}$ question : À quoi peut servir l'informatique ? Pour une secrétaire ; pour un musicien ; pou un balayeur ; pour le Père Noël ?

- $4^{\text {ème }}$ question : Dessine l'ordinateur de 1'an 2020.

Ces questions nous entraînent tout d'abord dans l'univers des genres scripturaux; quels types d'écrits ont été pratiqués dans ces ateliers, dans quelle mesure se rattachent-ils à la vie de l'enfant, de l'écolier, du futur citoyen ?

La question de l'outil d'écriture est également posée ainsi que celle des changements qu'il autorise. Il ne s'agit pas ici de changements décrits dans des pratiques mais des changements perçus par les élèves d'après leurs reflexions et leur expérience dans les ateliers. En extrayant des réponses à la troisième question les éléments relatifs à l'écriture, il est possible de dresser un 
tableau vivant de l'image que les enfants se font de l'écriture (au sens large) dans un certain monde du travail et de l'entreprise. La représentation par le dessin nous projette en avant puisqu'elle nous montre que le futur est, pour l'écolier d'auiourd'hui, placé assez nettement sous le signe de l'oral, de l'image et d'un nouvel équilibre entre l'oral et l'écrit.

2.1.1 Quel projet as-tu réalisé cette année avec ta classe à l'atelier informatique ?

Les réponses à cette question sont bien sûr liées aux projets d'atelier effectivement menés par les enfants durant l'année.

Le projet est souvent présenté avec un terme générique comme écriture de textes ou pratique du traitement de texte sans autre précision. Néanmoins un certain nombre de réponses sont plus précises et montrent que le genre le plus' représenté est le journal (145 citations), viennent ensuite la bande dessinée (22), la poésie (12), le conte ou l'histoire (12), le livre (10), l'album (6), des publicités (4), des " expositions" (5) et des rédactions (3). D'autres termes, en relation avec l'écrit sont plus vagues et plus proches de l'informatique : programme, raisonnement.

ex. : "Il y en a eu 2 : le ler nous avons été dans il etait une fois et nous avons créer l'histoire de notre choix puis nous avons fait. la mise en page et notre mâ̂tre l'a fait sortir sur l'imprimante (je parle du texte) le 2ème nous avons été dans orbiters 3 et nous avons fait un exposé sur la planète de notre choix, nous, avons fait de la mise en page et le mâ̂tre l'a fait sortir de l'imprimante" (note 4 ).

Le terme joumal est employé pour plusieurs sortes de textes différents ; essentiellement le journal de voyage, le journal de l'école, le journal d'informations qui évoque la presse adulte, le journal personnel. Après un voyage de nature en Allemagne les enfants d'un atelier constituent un journal à partir de textes documentaires et de textes de souvenirs personnels ; un autre atelier présente un joumal composé de plusieurs articles crées par des groupes différents.

ex. : "Un journal qui s'appelle " La dépêche des Naimbus " "

"Nous avons choisi de faire un journal qui comportait plusieurs sujets comme le cinéma, les petites annonces, le sport, l'actualité politique, est .. "

Les termes journal et album sont parfois employés l'un pour l'autre, exposition entre également en concurrence avec eux. A l'aide de logiciels spécialisés les enfants construisent des textes sur l'eau ou sur une planète de leur choix. C'est ici le mode de présentation et de diffusion qui diffère, plus que le produit

textuel lui-même. Les feuilles de l'album exposées sur des panneaux deviennent une exposition.

Les poèmes et les contes sont fréquemment illustrés grâce à un logiciel graphique et l'expression écrite est étroitement reliée à l'expression par l'image.

ex. : " des poèmes avec des dessins p logo, des affiches publicitaires "

" un texte et un château qu'on a pas terminé "

" nous avons fait le conte et son dessin" 
" 4 élèves ont fais un livre pour une classe de maternels et on a fait un journal sur l'école que l'on a vendu à la fête "

Les enfants évoquent également l'activité de résumé, qu'il s'agisse de résumer des textes documentaires issus de bases de données logicielles (planète, eau) ou d'écrire avec le traitement de texte des résumés d'ouvrages.

ex. : " nous avons écrit des résumés de livre comme 20000 lieux sous les mers, Alladin, le petit poucet, Pinocchio "

Il est question de bandes dessinées, de dessins animés, de la création d'une carte d'identité, de travaux concernant l'instruction civique.

ex. : "Nous avons commencé un dessin animé sur le western, nous avons fait une carte d'identité et des dessins "

Les genres évoqués jusqu'ici sont des genres scolaires d'écriture longue ou mi-longue ; parfois il semble que l'écriture ne soit qu'un prétexte à dessiner.

ex. : " on a fait sur l'ordinateur des phrases commensant par un mot et après il fallait faire un dessin "

Les textes écrits sont divers : récits de rêve, texte décrivant un monument, conte moyen-âgeux ... . Devant l'absence fréquente de spécification dans les réponses assez courtes des enfants il n'est pas toujours facile de se faire une idée des mondes signifiés dont relèvent ces textes ; ils appartiennent au monde réaliste (voyage, vie scolaire, vie politique), au monde du documentaire (eau, planètes, ordinateurs), au monde de fa fiction (bandes dessinées, contes) ou de l'imaginaire (récit de rêve, poésie, histoires).

Il n'y a pas à première vue de différence fondamentale entre des genres qui seraient abordés grâce à l'ordinateur et des genres qui apparâ̂traient dans un environnement papier/crayon. Seule l'évocation d'exercices en LOGO ou l'expérience de l'image animée nous montre que les enfants sont plongés dans l'univers informatique. La présence quasi constante du graphisme est également caractéristique du rapport à l'inscriptible dans ces ateliers par rapport à une pratique plus traditionnelle du français à l'école primaire. Ceci nous semble en relation avec la grande diffusion des écrits illustrés dans la littérature et de la presse enfantines.

Mais c'est pour la présentation des textes que le traitement de texte est le plus apprécié.

2.1.2. Qu'est-ce que l'ordinateur a rendu possible?

Pour ces jeunes, l'ordinateur permet avant tout d'écrire "plus facilement, plus correctement, au propre, avec les caractères qu'on veut, tous les caractères, en italique, en gras ", en tailles différentes et selon différents styles. Il est lié au plaisir du changement : " on peut changer l'écriture (plus gros, plus petit) ", le mode d'écriture. Les textes, romans, rédactions, sont gardés dans l'ordinateur ou sur une disquette plusieurs semaines, ce qui permet d'en remettre à plus tard l'impression. Certains se rendent compte qu'à l'avenir if faudra compter avec ce type d'écriture dans leur future activité professionnelle. 
ex. : "Faire des dessins, des textes, et grâce à l'ordinateur on avance plus vite dans les bureaux et dans les entreprises ".

D'autres ont déjà une vision très " robotique " et "domotique " des choses : l'ordinateur peut programmer l'allumage et l'extinction de la lumière, la mise en route de la cafetière, le départ d'une musique. Mais ceci nous éloigne du traitement de texte même si la programmation s'organise à partir de menus inscrits sur l'écran.

2.2. Que peux-tu faire avec un logiciel de traitement de texte que tu ne pourrais pas faire avec une machine à écrire?

Il ne faut pas attendre des réponses des enfants une "théorie naturelle " de l'écriture; les élèves ne donnent aucun renseignement sur leurs manières d'écrire un texte, sur leurs sources d'inspiration, leurs difficultés ; ils sont sensibles avant tout à la matérialité des objets techniques et à la facilité des différentes procédures permettant la diversification de l'écriture que nous venons d'observer dans les réponses à la précédente question.

Mises à part quelques rares réponses désabusées, naïves, "rien, des tas de choses, tout " ou peut-être illusoires "écrire plus long ", les enfants sont sensibles à un certain nombre d'actions rendues possibles du fait de l'informatisation. Ils sont heureux de ne plus être obligés de manipuler des feuilles, de devoir en changer, de retrouver au même endroit des textes écrits depuis plusieurs semaines. Outre l'intérêt de faire une mise en page agréable, ce sont les possibilités d'effacer, de sauvegarder, de modifier, de retourner en arrière, de dupliquer, qui les passionnent.

Une des procédures les plus souvent citées est l'effacement. Les enfants utilisent plusieurs termes pour en parler : gommer qui est un emprunt à l'univers papier/crayon, enlever, terme plus général, réparer, autre version de la correction qui articule l'opération d'effacement à celles de modification et d'amélioration.

ex. : " effacer une chose, modifier une autre sans avoir à tout recommencer"

" on peut revenir sur les fautes, on n'est pas obligé de changer de feuille."

Ces réponses qui impliquent l'idée de réécriture et de correction des textes ne préjugent en rien de la pratique effective de ces élèves ; mais elles signalent une prise en compte intéressée de la modification et de la révision (note 5$)$.

Certains ont déjà dû bénéficier de l'aide de correcteurs orthographiques et s'en trouver très heureux.

ex. : "le logiciel de traitement de texte peut corriger les fautes alos que la machine a ecrire ne peut pas. "

Enfin ta possibilité d'imprimer en différé et en de multiples exemplaires facilite la diffusion du texte, qu'elle soit interne à la classe, à l'école (pour les CP ou pour les maternels), ou externe (diffusion ou vente du journal 
de l'école à la fête de fin d'année). En ce sens, l'ordinateur " récupère " les fonctions de l'imprimerie popularisée dans les classes Freinet.

Plus que la facture du texte, c'est son aspect sémiotique qui est évoqué, sa fonction, son destinataire.

2.3. A quoi peut servir l'informatique? Pour une secrétaire, pour un musicien, pour un balayeur, pour le Père Noël?

La troisième question nous introduit, par le biais d'une question générale sur l'informatique, à l'utilisation du traitement de texte dans le monde du travail réel de quelques acteurs sociaux et dans celui, plus fantaisiste, du Père Noêl.

\subsubsection{La secrétaire}

Les enfants ont bien compris que la fonction de la secrétaire n'est plus seulement de prendre en note ce que dit son patron, de taper plus vite ses documents et ses textes mais également $d$ 'assurer des opérations de classement, de gestion, d'émission, de reception de messages au carrefour de la communication orale et de la communication écrite.

La nouvelle secrétaire garde en mémoire des informations, des noms, des adresses, des numéros de téléphone, elle inscrit les rendez-vous de son patron dans la partie agenda électronique de son ordinateur. Elle reçoit des informations, prépare ses enveloppes rapidement et expédie son courrier en mailing ; elle peut classer plus facilement des dossiers avant une réunion, les mémoriser ou les ranger en vue d'une prochaine utilisation. Son traitement de texte est lié à d'autres logiciels, tableurs, gestionnaires puisqu'elle peut s'occuper également de calculs divers, de commandes et même des fiches de paye du personnel. Les enfants ont tout à fait conscience de l'augmentation de la complexité de ce genre de poste de travail et des diverses opérations d'écriture, de classement et de comptage qu'il peut nécessiter. Nous retrouvons les différentes fonctions assumées par l'écriture telles qu'elles ont été décrites par l'ethnographie de l'écriture (Goody, 1977, Harris, 1993)".

ex. : "elle sert à écrire, à faire des dossiers pour le patron au lieu une douzaine de pages à la main. "

"A retenir des appels du travail, et envoyer des lettres vite faites grace

à l'ordinateur. "

81

\section{3.2.Le musicien et le balayeur}

Si les enfants sont majoritairement sûrs que les secrétaires ont besoin de l'informatique et plus particulièrement du traitement de texte, ils sont moins nombreux à répondre que ces outlfs présentent une utilité pour le musicien ou pour le balayeur. Néanmoins ceux qui répondent positivement le font avec astuce et imagination.

Ainsi le musicien utilise-t-il le traitement de texte pour écrire le texte de ses chansons. Il gère son emploi du temps et ses rendez-vous (les lieux et les jours où il donne ses concerts) au moyen de son agenda mais il a surtout des 
logiciefs spécialisés qui lui permettent de s'exercer à l'écriture musicale, de s'accompagner, d'enregistrer des musiques avec plusieurs timbres d'instruments et de tirer ensuite ses partitions sur son imprimante. L'amateur peut toujours, quant à lui, s'initier au solfège.

ex. : "l'ordinateur sert au musicien à écrire ses chansons ".

"trouver des notes, inventer des chansons et les enregistrer."

" â inscrire l'heure de ses concert."

Si l'ordinateur ne réussit pas à tirer le balayeur de son emploi par la consultation des offres d'emploi sur un réseau de type Minitel, il peut au moins lui permettre de rationaliser son travail, de calculer son salaire, de télécommander ses balais et ses voitures. Il lui permet de visualiser par exemple la carte de Paris, le plan du quartier où il doit intervenir, de construire un itinéraire et de noter au fur et à mesure l'avancée de son travail, les lieux et les jours d'intervention. L'ouvrier peut également savoir combien il a ramassé d'ordures, de poussières, combien il a vidé de poubelles et combien il lui reste de balais. A la pause, entre deux phases de gestion de ce qui devient véritablement une entreprise, le balayeur peut toujours utiliser son ordinateur pour jouer à des jeux vidéos !

ex. : "Pour savoir dans quel rue il va devoir aller balayer"

" à compter combien il a ramassé d'ordures" " à savoir les cartes de Paris"

\subsubsection{Le Père Noël}

C'est sans doute le Père Noêl qui se trouve à la tête du réseau le plus gigantesque. Quelques enfants ironisent et montrent leur scepticisme vis-à-vis de son existence ( "ah! ah! ah! ", "il n'existe pas "), mais la plupart jouent le jeu de la réponse. En effet, quoi de plus indispensable qu'un ordinateur pour permettre au Père Noël de noter tous les noms des enfants du monde, d'écrire le nom de ceux qui ne sont pas sages et qui n'auront pas de cadeau, d'enregistrer à la fois les souhaits des enfants et les cadeaux qu'il a déjà distribués. Il lui faut se tenir informé des derniers jouets fabriqué, " mettre en mémoire les jouets fabriqués, passer ses commandes "... et bien sûr faire ses comptes. Il possède heureusement un logiciel de reconnaissance de l'écriture "pour lire les écritures des enfants" ainsi qu' "un fax pour recevoir la liste de Jouets " ... Côté écriture, le Père Noêl est donc très organisé !

À travers ces portraits d'utilisateurs de l'ordinateur nous voyons apparâ̂tre une image de l'écriture sociale, liée aux outils technologiques les plus récents : réseaux télématiques, fax, logiciels adjacents au traitement de texte. En tant que collectif, ces élèves de CM2 semblent très au courant des possibilités d'émission et de réception à distance des messages écrits. Nous sommes ici dans le domaine de l'écrit fonctionnel, lié au listage, à la mise en tableaux, à la planification, écrits administratifs, commerciaux, à l'image d'une certaine face de notre société (note 6 ).

\subsection{Dessine 1'ordinateur de l'an 2020}


Si l'on s'en tient aux représentations graphiques les plus fréquentes, l'ordinateur du futur vu par les enfants est assez proche des standards actuels. Parfois beaucoup plus grand (mur entier), modulable, mais plutôt nettement plus petit (en porte-clefs, en montre, en baladeur ...), il comporte un écran, un clavier, une souris, des hauts-parleurs, une antenne. Il est souvent multimédia et possède parfois des fonctions et des accessoires surprenants : batte de base ball, appareil à faire cuire frites et hamburgers, à distribuer des boissons. L'enfant se représente parfois lui-même, seul, face à l'appareil qui devient une sorte de machine dispensatrice de tout ce dont il a besoin.

Si nous isolons les caractères qui sont encore peu représentés dans les ordinateurs actuellement commercialisés, nous voyons que l'aspect novateur le plus évident pour ces élèves réside dans la commande vocale et dans la transformation automatique de l'oral en écrit.

ex. : "On lui parlera et il fera ce qu'on lui dit"

"Tu parles et ça t'écris ce que ta dit."

L'ordinateur du futur sera parlant, reconnâ̂tra la "voix de son mâ̂tre " et inscrira docilement ce que celui-ci lui a dit sur son écran. Néanmoins il ne le libèrera pas pour autant de la lecture puisque, dès le matin, après une rapide salutation, il pourra afficher sur son écran le planning de la journée et demander des instructions,

ex. : "A FAIRE, tondre le gazon, faire le repas, passer l'aspirateur "

Le soir, en rentrant de l'école, l'élève attendra de son ordinateur qu'il lui fasse rapidement ses devoirs et qu'il obéisse à ses ordres (il pourra faire ce qu'il lui dit, dessiner ce qu'il tient à la main). Sur les dessins la taille de l'ordinateur est souvent réduite, le clavier peut être lui aussi réduit à quelques touches voire supprimé. C'est sur un clavier inscrit sur l'écran ou avec son crayon graphique que l'enfant pourra inscrire directement ses messages. Le dessin d'une fillette représente un petit ordinateur portable qui reconstitue en miniature une partie de ce que peut renfermer le lourd cartable d'une élève de CM2.

ex : "COMMANDE : stylo bleu / livre de géométrie / ROMAN / CLASSEUR "(note $7)$.

Cet instrument léger lui permet de recréer dans un espace réduit son bureau et ses outils familiers.

\section{POUR NE PAS CONCLURE}

Après un an d'initiation au traitement de texte, les enfants interrogés en juin 1994 avaient écrit des textes divers, journaux, poèmes, contes, B.D., etc. Ils avaient monté des expositions, fait des !ivres remplis d'histoires, animé des images.

Hormis les productions animées, il n'y a pas là de " révolution " apparente mais plutôt appropriation de l'outil ordinateur pour produire des écrits relativement traditionnels.

L'évolution nous parâ̂t se situer à d'autres niveaux. 
Tout d'abord au niveau de la conscientisation du processus d'écriture qui met en relation étroite processus d'initiation et processus d'expertise. Le logiciel de traitement de texte permet de présenter un produit d'acceptabilité minimale, qui n'est pas spécialement perçu comme enfantin (note 8 ). Il nécessite un passage obligé par une binarisation et une procéduralité des événements qui relèvent de l'organisation et de la planification des actions telles qu'elles sont envisagées par l'intelligence artificielle du moment. L'accent est mis sur la sémiotisation du produit fini.

Nous observons également une conscientisation de la place de l'écriture au sein d'activités sociales diverses liées au monde de l'entreprise : programmer, classer, calculer, gérer, communiquer. Pour l'enfant qui écrit sur l'ordinateur, il y a complexification et diversification des rôles et des postes de travail en un même lieu, tout dépend de la diversité des logiciels intégrés dans le disque dur de la machine et des périphériques branchés. L'écrivain devient typographe et imprimeur mais aussi documentaliste, graphiste ou même cinéaste. C'est d'ailleurs plus l'image du journaliste que celle de l'écrivain qui se profile en filigrane dans ces réponses au questionnaire.

Le scripteur n'est pas uniquement quelqu'un d'assujetti à une théorie du texte mais un sujet qui assume des rôles sociaux divers régulièrement attachés à la production des écrits : le reporter ou le documentaliste qui part en quête d'informations dans une base de données, le dessinateur qui utilise sa palette pour illustrer le texte du poète, le correcteur qui reprend les épreuves de l'essayiste ... Toutes ces personnalités peuvent se rencontrer à la confluence d'un lieu spatial, l'ordinateur, et d'un lieu psychique, l'élève ou le groupe d'élèves en poste devant l'appareil.

$84 \sim$

\section{NOTES}

1

Colloque de Cerisy(1991) : L'imagination informatique, PUV.

2

Notre recherche actuelle porte sur l'analyse des réponses aux questions ouvertes d'un questionnaire d'évaluation en informatique donné en juin 1994 à plusieurs centaines de jeunes de CM2 participant aux ateliers informatiques de 11 arrondissements de Paris $\left(1^{\mathrm{er}}, 2^{\text {ème }}, 3^{\text {ème }}, 4\right.$ ème $, 5^{\text {ème }}, 6^{\text {ème }}, 11^{\text {ème }}, 14^{\text {ème }}, 16^{\text {ème }}, 17^{\text {ème }}$, $\left.18^{\text {ème }}, 20^{\text {ème }}\right)$. Ces ateliers étaient équipés en matériel compatible PC, Macintosh ou Atari. Nous 'avons travaillé sur 409 questionnaires remplis. Nous avons utilisé pour la présentation rapide de cet article une partie du contenu des réponses aux questions $8,21,30$ et 34 . La recherche plus précisément linguistique qui nous préoccupe a trait aux liens lexico-syntaxiques et sémantiques entre les questions posées par l'équipe de formateurs adultes et les réponses des enfants. Dans le souci de ne pas alourdir la présentation, nous avons passé ces aspect sous silence.

3

Cf. la question 20 du questionnaire "quels logiciels as-tu utilisé cette année à l'atelier informatique ? " Nous relèverons les plus utilisés des traitements de texte et des logiciels graphiques : Word, WinWord, Publisher, Rédacteur, Redige3c, il était une fois, Wizard Paint,D Paintos, Paint Brush, Mac Paint, 'Deluxe Paint, Neochrom ... 
4

Dans la présentation des réponses en italiques nous respectons l'orthographe originale des enfants.

5

D'après les observations que nous avions faites en 1990 dans deux ateliers informatiques, les enfants ne pratiquaient que très peu la reécriture et la modification de leurs textes ; néanmoins écrivant de manière collective ils exprimaient à l'oral avant d'écrire : sur le clavier plusieurs versions potentielles du texte dont une seule était finalement actualisée (cf. Pouder 1992). La pratique de la rééducation du langage écrit montre également combien l'idée même de modifier unmessage déjà inscrit sur une feuille est intolérable pour certains enfants.

6

Cf. Goody, La raison graphique, p.248 : "J'accorde plus d'importance au tableau et à la liste. La liste est en relation directe et étroite avec les processus cognitifs. En effet, faire (réellement ou mentalement) ce genre de listes ... relève de ce processus plus général qu'est la planification de l'action ... on peut considérer que planifier c'est construire une liste des épreuves à accompli r... la liste est une représentation hiérarchique du comportement. Ce qui caractérise l'homme, ce n'est pas tant le fait de faire des plans, de recourir à la pensée symbolique que d'extérioriser, de communiquer ces plans, d'opérer des transactions portant sur ces produits de la pensée symbolique. Et c'est précisément ce genre d'activité qu'encourage, que transforme et même que transfigure l'écriture ".

7

Nous présentons en annexe trois exemples de dessins répondant à la consigne. 8

Les destinataires des logiciels d'écriture pour "enfants " sont sans doute plus les enfants en cours d'apprentissage de l'écriture (les 5-7 ans) que les enfants de 11 ans. Le pré-adolescent est ressenti comme un futur consommateur de la presse adulte, un co-utilisateur de l'ordinateur familial ; au delà des techniques il est confronté aux genres scripturaux de son époque.

\section{BIBLIOGRAPHIE}

BALPE J.-P., (1995), Littérature et poésie sur livre électronique, La Recherche, vol. 26 .

BRETON P., (1990), Une histoire de l'informatique. Paris, Seuil. COLLOQUE DE CERISY, (1991), L'imagination informatique de la littérature. Coll. L'imaginaire du texte, Presses Universitaire de Vincennes.

CNRS \& UNIVERSITES, (1995), L'Internet professionnel : Témoignages, expériences, conseils pratiQues de la communauté enseignement et recherche, LMB monographie $n^{\circ} 1$, CNRS éditions.

DIDIER B. et NEEFS J., (Dir., 1990), Penser, classer, écrire, de Pascal à Pérec. Coll. L'imaginaire du texte, Presses Universitaires de Vincennes.

GOODY J., (1977), La raison graphique, la domestication de la pensée sauvage, Paris, Le sens commun, Editions de Minuit.

HARRIS R., (1993), La sémiologie de l'écriture. Paris. CNRS Langage, CNRS éditions. 
LEVY P., (1987), La machine univers, création, cognition et culture informatique. Paris, La découverte, coll. Points Sciences.

LIEUX DE L'ENFANCE, (1985), L'enfant et l'ordinateur. Toulouse. Privat, $\mathrm{n}^{\circ} 4$.

MARKS GREENFIELD P., (1984), Mind and Media, The effects of television, computers and videogames, The Developing Chi!d, Editors Jerome Bruner Michael Core Barbara Lloyd, Fontana Papersbacks.

PAPERT S., (1981), Le jaillissement de l'esprit, ordinateurs et apprentissage. Paris, Flammarion.

PESSIS-PASTERNAK G. (entretiens avec), (1991), Faut-il brûler Descartes ? Du chaos à l'intelligence artificiell : quand les scientifiques s'interrogent. Paris, La découverte.

POUDER M.-Ch., (1992), Produire un récit à deux sur ordinateur : entre programmation de l'écriture et accident du dialogue, in Réécriture et interactivité en situation scolaire, Paris, CALAP $n^{\circ}$ 9, CNRS-Université René Descartes.

POUDER M.-Ch., (1993b), Influence de l'audiovisuel sur les productions orales et écrites d'élèves du CM2. Paris, INRP, Repères $\mathrm{n}^{\circ} 7$.

REPÈRES, (1991), Articulation oral/écrit. Paris, INRP, n³.

Annexes

Fig. 1

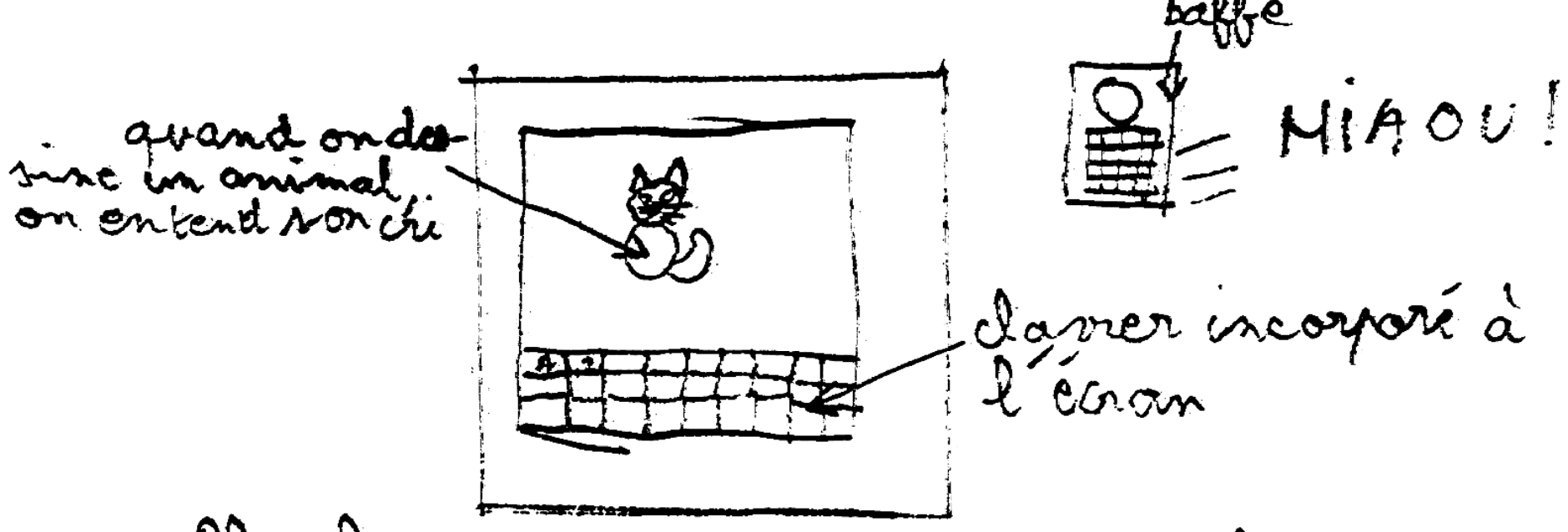

Plus bes-oin de Prancher los lits, il manche sur piles!

Fig. 2 


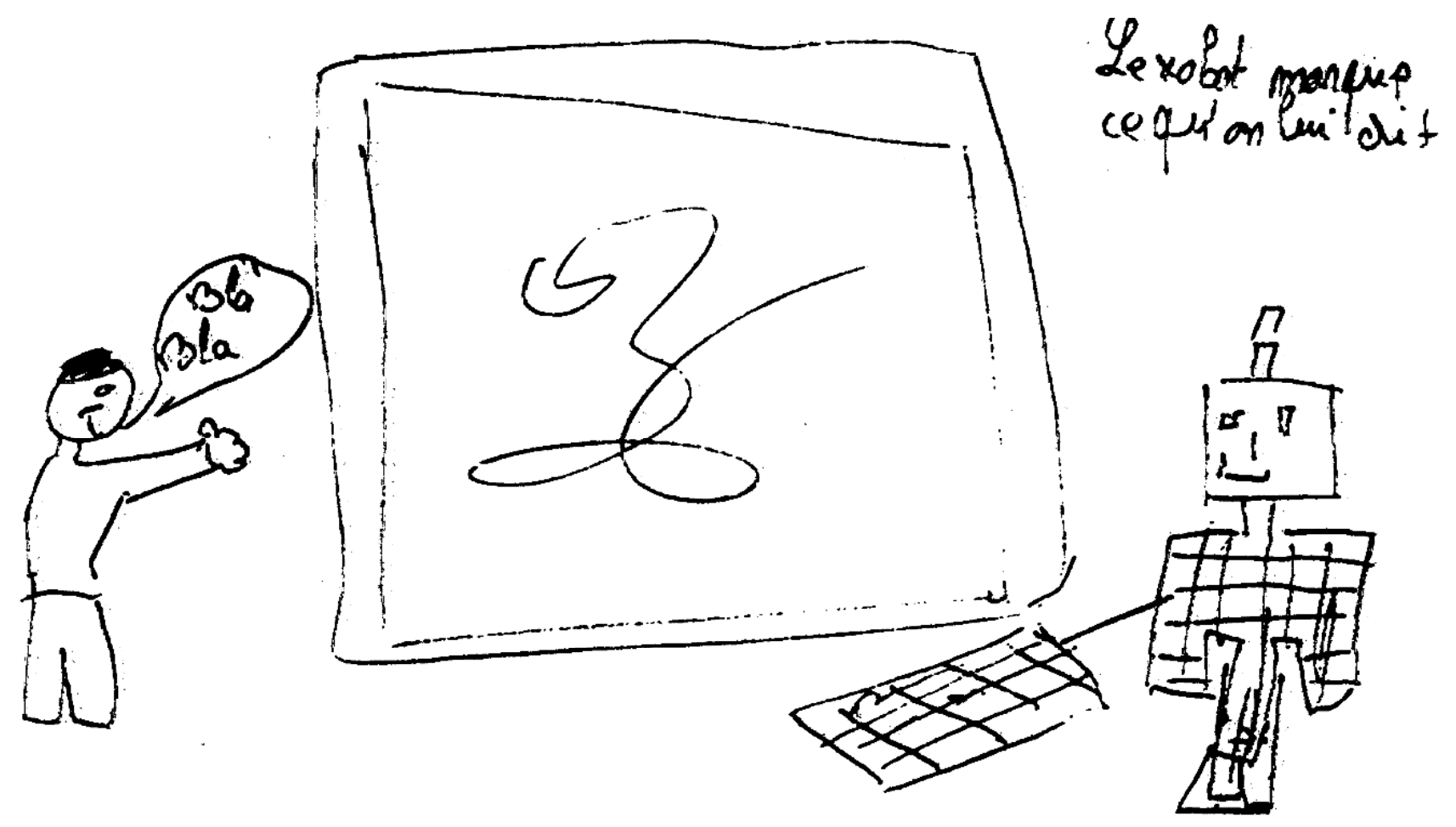

Fig. 3

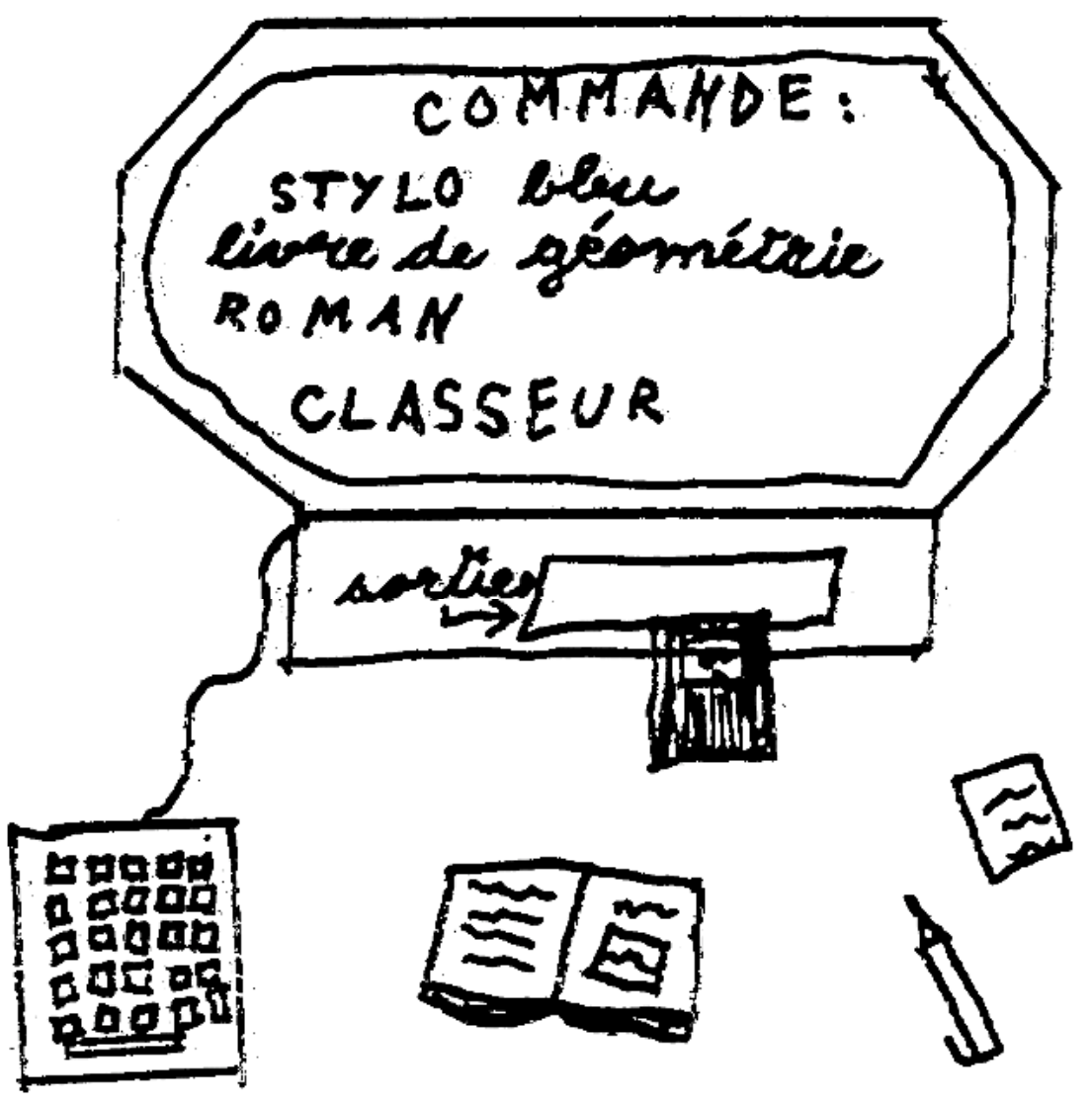

